\title{
CLIFFORD ALGEBRAS AND THE CLASSICAL DYNAMICAL YANG-BAXTER EQUATION
}

\author{
A. Alekseev and E. Meinrenken
}

\begin{abstract}
We describe a relationship of the classical dynamical Yang-Baxter equation with the following elementary problem for Clifford algebras: Given a vector space $V$ with quadratic form $\mathcal{Q}_{V}$, how is the exponential of an element in $\wedge^{2}(V)$ under exterior algebra multiplication related to its exponential under Clifford multiplication?
\end{abstract}

\section{Introduction}

Let $\mathfrak{g}$ be a real Lie algebra, equipped with a non-degenerate invariant quadratic form $\mathcal{Q}$. Let $\Theta \in \wedge^{3} \mathfrak{g}$ be the cubic element defined by the quadratic form and the Lie algebra structure. An element $\mathfrak{r} \in \wedge^{2} \mathfrak{g}$ is called a classical $r$-matrix for $\mathfrak{g}$ if it satisfies the (modified) classical Yang-Baxter equation (CYBE)

$$
\frac{1}{2}[\mathfrak{r}, \mathfrak{r}]^{\mathfrak{g}}=\epsilon \Theta
$$

for some coupling constant $\epsilon \in \mathbb{R}$. Here $[\mathfrak{r}, \mathfrak{r}]^{\mathfrak{g}}$ is defined using the extension of the Lie bracket to the Schouten bracket on the exterior algebra, $[\cdot, \cdot]^{\mathfrak{g}}: \wedge^{k} \mathfrak{g} \times$ $\wedge^{l} \mathfrak{g} \rightarrow \wedge^{k+l-1} \mathfrak{g}$. Drinfeld [8] and Semenov-Tian-Shansky [20] gave a geometric interpretation of the CYBE in terms of Poisson-Lie group structures, and a classification of $r$-matrices for semi-simple Lie algebras and $\epsilon \neq 0$ was obtained by Belavin-Drinfeld [6].

The CYBE admits an important generalization known as the classical dynamical Yang-Baxter equation (CDYBE). Let $\mathfrak{k} \subset \mathfrak{g}$ be a Lie subalgebra. A classical dynamical r-matrix is a $\mathfrak{k}$-equivariant (meromorphic) function $\mathfrak{r}: \mathfrak{k}^{*} \rightarrow \wedge^{2} \mathfrak{g}$ satisfying the (modified) CDYBE [11]

$$
\sum_{i} \frac{\partial \mathfrak{r}}{\partial \mu_{i}} \wedge e_{i}+\frac{1}{2}[\mathfrak{r}, \mathfrak{r}]^{\mathfrak{g}}=\epsilon \Theta
$$

Here $e_{i}$ is a basis on $\mathfrak{k}$ with dual basis $e^{i} \in \mathfrak{k}^{*}$, and $\mu_{i}$ are the corresponding coordinates on $\mathfrak{k}^{*}$.

The CDYBE was formulated for $\mathfrak{g}$ semi-simple and $\mathfrak{k}$ a Cartan subalgebra in a 1990 paper by Balog-Dabrowski-Feher [4, page 231], who also described the basic trigonometric solution for this case. Etingof-Varchenko [11] interpreted classical dynamical r-matrices in terms of Poisson Lie groupoids, and gave a

Received September 23, 2002. 
classification for $\mathfrak{g}$ semi-simple and $\mathfrak{k}$ of maximal rank. The classification was extended by Schiffmann [19] to more general subalgebras, interpolating between the Belavin-Drinfeld $(\mathfrak{k}=0)$ and the Etingof-Varchenko cases. For more general $\mathfrak{g}$, Etingof-Schiffmann [10] introduced the moduli space of classical dynamical $r$-matrices, and described its structure.

The CDYBE was discovered in conformal field theory and the theory of quantum groups $[4,5,13]$, but arises in many other contexts as well. Fehér-GáborPusztai [12] describe their appearance in Dirac reduction. Poisson geometric applications include work by Jiang-Hua Lu [16], who proved that Poisson homogeneous structures on $G / T$ (for a compact Lie group $G$ with maximal torus $T$ ) all come from solution of the CDYBE, and Ping $\mathrm{Xu}[21]$ who showed that solutions of the CDYBE for $(\mathfrak{g}, \mathfrak{k})$ give rise to certain invariant Poisson structures on $\mathfrak{k}^{*} \times G$. The CDYBE also appears in the theory of quasi-Poisson manifolds [1] and is related to the Duflo map in Lie theory [3]. Petracci [18] has studied the CDYBE for arbitrary Lie algebras, and found relations to Lie's third theorem and the Poincaré-Birkhoff-Witt isomorphism.

Our goal in this paper is to explain a close relationship of the CDYBE with the following elementary problem in the theory of Clifford algebras. Suppose $V$ is a vector space with a quadratic form $\mathcal{Q}_{V}$, and let $\mathrm{Cl}(V)$ be its Clifford algebra. Recall that there is a vector space isomorphism $q: \wedge V \rightarrow \mathrm{Cl}(V)$, called the quantization map, and that elements $q\left(\wedge^{2} V\right) \subset \mathrm{Cl}(V)$ exponentiate to the Spin group $\operatorname{Spin}(V) \subset \mathrm{Cl}(V)$. One can then ask: How is the exponential $\exp (q(\lambda)) \in$ $\mathrm{Cl}(V)$ of $\lambda \in \wedge^{2}(V)$ related to the corresponding exponential $\exp (\lambda) \in \wedge(V)$ in the exterior algebra? Clifford calculus gives nice formulas for this and closely related problems. Taking $V=\mathfrak{g}$, the answer to this problem produces solutions of the CDYBE.

\section{Clifford exponentials}

In this section we describe various formulas for exponentials of quadratic elements in a Clifford algebra. Proofs of these formulas will be given in Section 5 below.

Let $V$ be a finite-dimensional real vector space, equipped with a nondegenerate quadratic form $\mathcal{Q}_{V}$. The pair $\left(V, \mathcal{Q}_{V}\right)$ will be called a quadratic vector space. The Clifford algebra $\mathrm{Cl}(V)$ is the quotient of the tensor algebra $\mathcal{T}(V)$ by the ideal generated by elements $v \otimes v-\frac{1}{2} \mathcal{Q}(v), v \in V$.

The involutive automorphism $a \in \operatorname{Aut}(\mathrm{Cl}(V))$ given on generators by $a(v)=$ $-v$ gives $\mathrm{Cl}(V)$ the structure of a $\mathbb{Z}_{2}$-graded algebra. For the rest of this paper, commutators in the Clifford algebra, tensor products with other $\mathbb{Z}_{2}$-graded algebras and so on will always be taken in the $\mathbb{Z}_{2}$-graded sense. Let

$$
q: \wedge V \rightarrow \mathrm{Cl}(V)
$$

be the quantization map, defined by the inclusion $\wedge V \rightarrow \mathcal{T}(V)$ as anti-symmetric tensors followed by the quotient map $\mathcal{T}(V) \rightarrow \mathrm{Cl}(V)$. The quantization map $q$ is an isomorphism of vector spaces, with inverse $q^{-1}$ the symbol map. 
Let $\mathrm{O}(V)$ denote the orthogonal group of $\left(V, \mathcal{Q}_{V}\right)$, o $(V)$ its Lie algebra, and

$$
\lambda: \mathrm{o}(V) \rightarrow \wedge^{2} V, \quad \lambda(A)=\frac{1}{2} \sum_{a} A\left(e_{a}\right) \wedge e^{a}
$$

the canonical isomorphism. Here $e_{a}$ is a basis of $V$ with dual basis $e^{a} \in V^{*}$ (identified with $V$ via $\mathcal{Q}_{V}$ ). The map

$$
\gamma: \mathrm{o}(V) \rightarrow \mathrm{Cl}(V), \quad \gamma(A)=q(\lambda(A))
$$

is a Lie algebra homomorphism into the even part of the Clifford algebra (with bracket the commutator). $\gamma(A)$ generates the action of $A$ as a derivation of $\mathrm{Cl}(V)$, that is,

$$
A(v)=[\gamma(A), v]
$$

for all $v \in V \subset \mathrm{Cl}(V)$. We will be interested in formulas for the Clifford algebra exponential $\exp (\gamma(A))$. One such formula reads (cf. [7, Proposition 3.13])

$$
q^{-1}(\exp (\gamma(A)))=\operatorname{det}^{1 / 2}(\cosh (A / 2)) \exp (2 \lambda(\tanh (A / 2))),
$$

where the square root of the determinant is a well-defined analytic function of $A$, equal to 1 at $A=0$. If $\operatorname{dim} V$ is even, one has an alternative expression

$$
\left.q^{-1}(\exp (\gamma(A)))=\operatorname{det}^{1 / 2}(2 \sinh (A / 2)) \exp \left(\iota_{\frac{1}{2} \lambda(\operatorname{coth}(A / 2)}\right)\right) \mathrm{d} \text { Vol }
$$

Here $\mathrm{d} \mathrm{Vol}$ is the Euclidean volume form on $V$, for a given choice of orientation, and the square root of the determinant is defined as a Pfaffian. More generally, given commuting elements $c \in \mathrm{O}(V)$ and $A \in \mathrm{o}(V)$, and a lift $\hat{c} \in \operatorname{Pin}(V)$ one has the formula

$$
q^{-1}(\hat{c} \exp (\gamma(A)))= \pm \operatorname{det}^{1 / 2}(c \exp (A)-I) \exp \left(\iota_{\frac{1}{2} \lambda\left(\frac{c \exp (A)+I}{c \exp (A)-I}\right)}\right) \mathrm{d} \operatorname{Vol},
$$

where the sign depends on the choice of lift.

Most important for our purposes will be a formula relating $\exp (\gamma(A))$ to the corresponding exponential $\exp (\lambda(A))$ in the exterior algebra. Consider the holomorphic function

$$
j(z)=\frac{\sinh (z / 2)}{z / 2}
$$

and let $f(z)$ be its logarithmic derivative,

$$
f(z)=(\ln j)^{\prime}(z)=\frac{1}{2} \operatorname{coth}\left(\frac{z}{2}\right)-\frac{1}{z} .
$$

Note that $j$ is symmetric with simple zeroes at points $z \in 2 \pi \sqrt{-1} \mathbb{Z} \backslash\{0\}$, while $f$ is anti-symmetric with simple poles at those points. The function $J \in C^{\infty}(\mathrm{o}(V))$ given by

$$
J(A)=\operatorname{det}(j(A))
$$

admits a unique smooth square root equal to 1 at $A=0$. Define a meromorphic function $\mathfrak{r}: \mathrm{o}(V) \rightarrow \wedge^{2}(V)$ by

$$
\mathfrak{r}(A)=\lambda(f(A))
$$


and set $\mathcal{S}: \mathrm{o}(V) \rightarrow \wedge^{\text {even }}(V)$,

$$
\mathcal{S}(A)=J^{1 / 2}(A) \exp (\mathfrak{r}(A)) .
$$

Theorem 2.1. The function $\mathcal{S}$ is analytic on all of $\mathrm{o}(V)$. Let $E$ be a vector space of "parameters", and $\phi: V \rightarrow E$ a linear map with components $\phi^{a}=$ $\phi\left(e^{a}\right)$. For all $A \in \mathrm{o}(V)$, the following identity holds in $\mathrm{Cl}(V) \otimes \wedge(E)$ :

$$
q \circ \iota_{\mathcal{S}(A)} \exp \left(\lambda(A)-\sum_{a} e_{a} \phi^{a}\right)=\exp \left(\gamma(A)-\sum_{a} e_{a} \phi^{a}\right) .
$$

Notice that if $A$ has no eigenvalue equal to 0 so that $\lambda(A) \in \wedge^{2}(V)$ is nondegenerate, any element $\alpha \in \wedge(V)$ can be written in the form $\alpha=\iota_{\beta} e^{\lambda(A)}$ for a unique element $\beta \in \wedge(V)$. This explains formula (6) for $\phi=0$ and $\lambda(A)$ nondegenerate. The remarkable feature of this formula is that $\mathcal{S}$ extends analytically to all $A$, and is independent of $\phi$.

For applications to Lie algebras, it is sometimes useful to write the right hand side of (6) somewhat differently. Define holomorphic functions

$$
g(z)=\frac{\sinh (z)-z}{z^{2}}, \quad j^{R}(z)=\frac{e^{z}-1}{z}, \quad j^{L}(z)=\frac{1-e^{-z}}{z} .
$$

Let $\psi=\phi \circ j^{R}(A): V \rightarrow E$, with components $\psi^{a}=\psi\left(e^{a}\right)$, and let $\varpi(A) \in \wedge^{2}(E)$ be the image of $\lambda(g(A)) \in \wedge^{2}(V)$ under the extended map $\phi: \wedge(V) \rightarrow \wedge(E)$. Then

$$
q \circ \iota_{\mathcal{S}(A)} \exp \left(\lambda(A)-\sum_{a} e_{a} \phi^{a}\right)=\exp (-\varpi(A)) \exp (\gamma(A)) \exp \left(-\sum_{a} e_{a} \psi^{a}\right) .
$$

\section{Quadratic Lie algebras}

In this Section, we specialize Theorem 2.1 to Lie algebras with an invariant quadratic form. We show that the exponentials in the Clifford and exterior algebras satisfy natural differential equations. In the following Section the dynamical Yang-Baxter equation emerges as a consistency condition for these differential equations.

3.1. Lie algebra consequences of Theorem 2.1. A quadratic Lie algebra is a Lie algebra $\mathfrak{g}$, together with an invariant, non-degenerate quadratic form $\mathcal{Q}$. We will denote the Lie bracket by $[\cdot, \cdot]^{\mathfrak{g}}$, to avoid confusion with commutators. The invariance condition means that the adjoint representation ad $: \mathfrak{g} \rightarrow \operatorname{End}(\mathfrak{g})$ takes values in $o(\mathfrak{g})$. Examples of quadratic Lie algebras include semi-simple Lie algebras, and semi-direct products $\mathfrak{g}=\mathfrak{s} \ltimes \mathfrak{s}^{*}$, where $\mathfrak{s}$ is any real Lie algebra, acting on its dual $\mathfrak{s}^{*}$ by the coadjoint action. Also, given a possibly degenerate invariant quadratic form $\mathcal{Q}^{\prime}$ on a Lie algebra $\mathfrak{g}^{\prime}$, the quotient of $\mathfrak{g}=\mathfrak{g}^{\prime} / K$ by the radical $K$ of the quadratic form is a quadratic Lie algebra. Note that quadratic Lie algebras are unimodular. See the work of Medina-Revoy [17] for further information and classification results.

The ingredients in Theorem 2.1, and its consequences, take on geometric meanings if $V=\mathfrak{g}$ is a quadratic Lie algebra, and $A=\operatorname{ad}_{\mu}$ for $\mu \in \mathfrak{g}$. 
$\lambda(A), \gamma(A)$ The map $\lambda^{\mathfrak{g}}=\lambda \circ$ ad $: \mathfrak{g} \rightarrow \wedge^{2} \mathfrak{g}$ is the map dual to the Lie bracket. The map $\gamma^{\mathfrak{g}}=q\left(\lambda^{\mathfrak{g}}\right): \mathfrak{g} \rightarrow \mathrm{Cl}(\mathfrak{g})$ generates the adjoint action of $\mathfrak{g}$ on the Clifford algebra.

$j^{L / R}(A)$ Let $G$ be the connected, simply connected Lie group having $\mathfrak{g}$ as its Lie algebra, and let $\exp _{G}: \mathfrak{g} \rightarrow G$ be the exponential map. Denote by $\theta^{L}, \theta^{R} \in \Omega^{1}(G, \mathfrak{g})$ the left/right invariant Maurer-Cartan forms. Under left trivialization of the tangent bundle $T G$, the differential $\exp _{G}$ at $\mu \in \mathfrak{g}$ is given by the operator $j^{L}\left(\operatorname{ad}_{\mu}\right)$ (cf. [14, Theorem II.1.7]). Equivalently, the value of $\exp ^{*} \theta^{L}$ at $\mu$ is given by

$$
\left(\exp ^{*} \theta^{L}\right)_{\mu}=j^{L}\left(\operatorname{ad}_{\mu}\right) .
$$

Similarly one has $\left(\exp ^{*} \theta^{R}\right)_{\mu}=j^{R}\left(\operatorname{ad}_{\mu}\right)$.

$J(A)$ Let $J^{\mathfrak{g}}=J \circ$ ad. The quadratic form $\mathcal{Q}$ defines a translation invariant measure on $\mathfrak{g}$ and a bi-invariant measure on $G$. Since $\mathfrak{g}$ is unimodular, $\operatorname{det}\left(j^{L}\left(\operatorname{ad}_{\mu}\right)\right)=\operatorname{det}\left(j^{R}\left(\operatorname{ad}_{\mu}\right)\right)=J^{\mathfrak{g}}(\mu)$. Thus $J^{\mathfrak{g}}$ is the Jacobian of the exponential map $\exp _{G}$ with respect to left or right trivialization of $T G$, and the subset of $\mathfrak{g}$ where $A=\operatorname{ad}_{\mu}$ has eigenvalues in $2 \pi i \mathbb{Z} \backslash\{0\}$ is the set of critical points.

$\varpi(A)$ Let $B\left(\mu, \mu^{\prime}\right)=\frac{1}{2}\left(\mathcal{Q}\left(\mu+\mu^{\prime}\right)-\mathcal{Q}(\mu)-\mathcal{Q}\left(\mu^{\prime}\right)\right)$ denote the symmetric bilinear form associated with $\mathcal{Q}$. Let $\eta=\frac{1}{12} B\left(\theta^{L},\left[\theta^{L}, \theta^{L}\right]^{\mathfrak{g}}\right) \in \Omega^{3}(G)$ be the Cartan 3-form on $G$. It is bi-invariant and therefore closed. Let $\varpi^{\mathfrak{g}} \in \Omega^{2}(\mathfrak{g})$ be the image of $\exp _{G}^{*} \eta$ under the usual homotopy operator $\Omega^{p}(\mathfrak{g}) \rightarrow \Omega^{p-1}(\mathfrak{g})$, so that $\mathrm{d} \varpi^{\mathfrak{g}}=\exp _{G}^{*} \eta$. If we identify $\wedge^{2} T_{\mu}^{*} \mathfrak{g} \cong \wedge^{2} \mathfrak{g}$, the value of $\varpi^{\mathfrak{g}}$ at $\mu$ is given by the formula (cf. [2]),

$$
\varpi_{\mu}^{\mathfrak{g}}=\varpi\left(\operatorname{ad}_{\mu}\right) .
$$

$\mathfrak{r}(A)$ Let $\mathfrak{r}^{\mathfrak{g}}(\mu)=\mathfrak{r}\left(\operatorname{ad}_{\mu}\right)=\lambda\left(f\left(\operatorname{ad}_{\mu}\right)\right)$. Given $\xi \in \mathfrak{g}$ let $\xi^{L}, \xi^{R}$ denote the left/right invariant vector fields on $G$ generated by $\xi$. On the subset of $\mathfrak{g}$ where $\exp _{G}$ is regular, the vector field $\frac{1}{2} \exp _{G}^{*}\left(\xi^{L}+\xi^{R}\right)$ is well-defined. It differs from the constant vector field $\xi$ by a vector field tangent to orbit directions for the adjoint action. It turns out [3, Lemma A.1] that the difference at $\mu \in \mathfrak{g}$ coincides with the vector field generated by $f\left(\operatorname{ad}_{\mu}\right) \xi$.

Theorem 2.1 takes on the following form. Let $\mathcal{S}^{\mathfrak{g}}(\mu)=\mathcal{S}\left(\operatorname{ad}_{\mu}\right)$.

Proposition 3.1. The identity

$$
q \circ \iota_{\mathcal{S}}\left(e^{\lambda^{\mathfrak{g}}-\Sigma_{a} e_{a} \phi^{a}}\right)=e^{\gamma^{\mathfrak{g}}-\Sigma_{a} e_{a} \phi^{a}}
$$

holds in $\mathrm{Cl}(\mathfrak{g}) \otimes C^{\infty}(\mathfrak{g}) \otimes \wedge E$.

The alternative formula (7) takes on a particularly nice form for $E=T_{\mu}^{*} \mathfrak{g}$, with $\phi: \mathfrak{g} \cong \mathfrak{g}^{*} \rightarrow T_{\mu}^{*} \mathfrak{g}$ the standard identification. Then $\phi^{a}=\mathrm{d} \mu^{a}$ where $\mu^{a}$ are the coordinate functions on $\mathfrak{g}$, and $\psi^{a}=\exp _{G}^{*}\left(\theta^{L}\right)^{a}$. Since $G$ is simply connected, the Lie algebra homomorphism $\gamma^{\mathfrak{g}}: \mathfrak{g} \rightarrow \mathrm{Cl}(\mathfrak{g})$ exponentiates to a 
Lie group homomorphism $\tau: G \rightarrow \operatorname{Spin}(\mathfrak{g})$, with $e^{\gamma^{\mathfrak{g}}}=\exp _{G}^{*} \tau$. The resulting formula

$$
q \circ \iota_{\mathcal{S} \mathfrak{g}}\left(e^{\lambda^{\mathfrak{g}}-\Sigma_{a} e_{a} d \mu^{a}}\right)=e^{-\varpi^{\mathfrak{g}}} \exp _{G}^{*}\left(\tau e^{-\Sigma_{a} e_{a}\left(\theta^{L}\right)^{a}}\right)
$$

relates $\tau e^{-\Sigma_{a} e_{a}\left(\theta^{L}\right)^{a}} \in \mathrm{Cl}(\mathfrak{g}) \otimes \Omega(G)$ and $e^{\lambda^{\mathfrak{g}}-\Sigma_{a} e_{a} d \mu^{a}} \in \wedge \mathfrak{g} \otimes \Omega(\mathfrak{g})$. For the case of compact Lie algebras, this result was proved in [3, Section 6.3].

3.2. Lie algebra differential. In this Section, $\mathfrak{g}$ denotes an arbitrary Lie algebra (not necessarily quadratic), and $\lambda^{\mathfrak{g}}: \mathfrak{g}^{*} \rightarrow \wedge^{2} \mathfrak{g}^{*}$ the map dual to the Lie bracket,

$$
\iota_{\xi} \iota_{\eta} \lambda^{\mathfrak{g}}(\mu)=\left\langle\mu,[\xi, \eta]^{\mathfrak{g}}\right\rangle .
$$

In a basis $e_{a}$ of $\mathfrak{g}$, with dual basis $e^{a}$ of $\mathfrak{g}^{*}$,

$$
\lambda^{\mathfrak{g}}\left(e^{c}\right)=-\frac{1}{2} \sum_{a b}^{c} f_{a b}^{c} e^{a} \wedge e^{b}
$$

where $f_{a b}^{c}=\left\langle e^{c},\left[e_{a}, e_{b}\right]^{\mathfrak{g}}\right\rangle$ are the structure constants. Recall that the Lie algebra differential $\mathrm{d}^{\mathfrak{g}}: \wedge^{\bullet} \mathfrak{g}^{*} \rightarrow \wedge^{\bullet+1} \mathfrak{g}^{*}$ is the (degree +1$)$ derivation given on generators $\mu \in \mathfrak{g}^{*}$ by

$$
\mathrm{d}^{\mathfrak{g}} \mu=\lambda^{\mathfrak{g}}(\mu) .
$$

Lemma 3.2. Let $E$ be some vector space, $\phi: \mathfrak{g} \rightarrow E$ a linear map, and $\phi_{a}=$ $\phi\left(e_{a}\right)$. Then

$$
\lambda^{\mathfrak{g}}-\sum_{a} e^{a} \phi_{a}
$$

is closed under the differential, $d^{\mathfrak{g}}+\sum_{a} \frac{\partial}{\partial \mu_{a}} \phi_{a}$ on $C^{\infty}\left(\mathfrak{g}^{*}\right) \otimes \wedge \mathfrak{g}^{*} \otimes \wedge E$.

Proof. Since $\mathrm{d}^{\mathfrak{g}} \lambda^{\mathfrak{g}}(\mu)=\mathrm{d}^{\mathfrak{g}} \mathrm{d}^{\mathfrak{g}} \mu=0$, this follows from the calculation,

$$
\mathrm{d}^{\mathfrak{g}}\left(\sum_{a} e^{a} \phi_{a}\right)=\sum_{a} \lambda^{\mathfrak{g}}\left(e^{a}\right) \phi_{a}=\sum_{a} \frac{\partial \lambda^{\mathfrak{g}}}{\partial \mu_{a}} \phi_{a} .
$$

We will need the following Lemma, describing the transformation of $\mathrm{d}^{\mathfrak{g}}$ under conjugation with $\exp \left(\iota_{\mathfrak{r}}\right)$ :

Lemma 3.3. For any $\mathfrak{r} \in \wedge^{2} \mathfrak{g}$,

$$
\exp \left(-\iota_{\mathfrak{r}}\right) \circ d^{\mathfrak{g}} \circ \exp \left(\iota_{\mathfrak{r}}\right)=d^{\mathfrak{g}}-\frac{1}{2} \iota_{[\mathfrak{r}, \mathfrak{r}] \mathfrak{g}}+\sum_{a} e^{a} \circ \iota_{\left[e_{a}, \mathfrak{r}\right] \mathfrak{g}}-\iota_{\mathfrak{u}}
$$

where $\mathfrak{u} \in \mathfrak{g}$ is the image of $\mathfrak{r}$ under the Lie bracket map $\wedge^{2} \mathfrak{g} \rightarrow \mathfrak{g}, \xi \wedge \xi^{\prime} \mapsto\left[\xi, \xi^{\prime}\right]^{\mathfrak{g}}$.

Proof. Write $\iota_{a}=\iota_{e_{a}}$. Then $\mathrm{d}^{\mathfrak{g}}=-\frac{1}{2} \sum_{a b c} f_{a b}^{c} e^{a} \wedge e^{b} \circ \iota_{c}$. Introduce components $\mathfrak{r}^{a b}$ by $\mathfrak{r}=\frac{1}{2} \sum_{a b} \mathfrak{r}^{a b} e_{a} \wedge e_{b}$. Then the Schouten bracket of $\mathfrak{r}$ with itself is given by the formula,

$$
[\mathfrak{r}, \mathfrak{r}]^{\mathfrak{g}}=\sum_{a b c}\left(\sum_{k l} \mathfrak{r}^{a k} f_{k l}^{b} \mathfrak{r}^{l c}\right) e_{a} \wedge e_{b} \wedge e_{c}
$$


We compute the left hand side of $(11)$ as a $\operatorname{sum} \sum_{j=0}^{\infty} \frac{1}{j !} \operatorname{ad}^{j}\left(-\iota_{\mathfrak{r}}\right) \mathrm{d}^{\mathfrak{g}}$ :

$$
\begin{aligned}
\operatorname{ad}\left(-\iota_{\mathfrak{r}}\right) \mathrm{d}^{\mathfrak{g}} & =\frac{1}{4} \sum_{a b k l m} \mathfrak{r}^{a b} f_{l m}^{k}\left[\iota_{a} \iota_{b}, e^{l} \wedge e^{m}\right] \iota_{k} \\
& =-\sum_{a b k m} \mathfrak{r}^{a b} f_{b m}^{k} e^{m} \iota_{a} \iota_{k}-\frac{1}{2} \sum_{a b k} \mathfrak{r}^{a b} f_{a b}^{k} \iota_{k} \\
& =\sum_{a} e^{a} \circ \iota_{\left[e_{a}, \mathfrak{r}\right]}-\iota_{\mathfrak{u}}, \\
\operatorname{ad}^{2}\left(-\iota_{\mathfrak{r}}\right) \mathrm{d}^{\mathfrak{g}} & =\frac{1}{2} \sum_{a b k m s t} \mathfrak{r}^{s t} \mathfrak{r}^{a b} f_{b m}^{k}\left[\iota_{s} \iota_{t}, e^{m}\right] \iota_{a} \iota_{k}=\sum_{a b k s t} \mathfrak{r}^{a b} \mathfrak{r}^{s t} f_{b t}^{k} \iota_{s} \iota_{a} \iota_{k} \\
\operatorname{ad}^{m}\left(-\iota_{\mathfrak{r}}\right) \mathrm{d}^{\mathfrak{g}} & =0, \quad m \geq 3
\end{aligned}
$$

3.3. Clifford differential. Let $(\mathfrak{g}, \mathcal{Q})$ be a quadratic Lie algebra. Similar to (10) there is a unique odd derivation $\delta^{\mathfrak{g}}$ on $\mathrm{Cl}(\mathfrak{g})$ given on generators $\mu \in \mathfrak{g}$ by

$$
\delta^{\mathfrak{g}} \mu=\gamma^{\mathfrak{g}}(\mu) .
$$

In fact $\delta^{\mathfrak{g}}$ may be written as a $\left(\mathbb{Z}_{2}\right.$-graded) commutator: Let $\Theta \in \wedge^{3} \mathfrak{g}$ be the cubic element defined by $\iota_{\mu} \Theta=\lambda^{\mathfrak{g}}(\mu)$. In terms of a basis $e_{a}$ of $\mathfrak{g}$, with dual basis $e^{a}$, we have

$$
\Theta=-\frac{1}{6} \sum_{a b c} f^{a b c} e_{a} \wedge e_{b} \wedge e_{c}
$$

where $f^{a b c}=B\left(e^{a},\left[e^{b}, e^{c}\right]^{\mathfrak{g}}\right)$. Then $\delta^{\mathfrak{g}}=[q(\Theta), \cdot]$. Kostant-Sternberg [15] made the beautiful observation that $q(\Theta)$ squares to a constant, hence that $\delta^{\mathfrak{g}}$ squares to 0 . We will call $\delta^{\mathfrak{g}}$ the Clifford differential. Under the quantization map $q$, the Lie algebra and Clifford differentials are related as follows [3, Proposition 3.3]:

$$
q^{-1} \circ \delta^{\mathfrak{g}} \circ q=\mathrm{d}^{\mathfrak{g}}+\frac{1}{4} \iota_{\Theta} .
$$

Replacing $\lambda$ with $\gamma$ in the proof of Lemma 3.2, we find:

Lemma 3.4. Let $E$ be some vector space, $\phi: \mathfrak{g} \rightarrow E$ a linear map, and $\phi^{a}=$ $\phi\left(e^{a}\right)$. Then

$$
\gamma^{\mathfrak{g}}-\sum_{a} e_{a} \phi^{a}
$$

is closed under the differential, $\delta^{\mathfrak{g}}+\sum_{a} \frac{\partial}{\partial \mu^{a}} \phi^{a}$ on $C^{\infty}(\mathfrak{g}) \otimes \mathrm{Cl}(\mathfrak{g}) \otimes \wedge E$.

\section{Solutions of the classical dynamical Yang-Baxter equation}

In this section, we will use our Clifford algebra techniques to construct solutions to the CDYBE in a number of cases.

Theorem 4.1. The function $\mathfrak{r}^{\mathfrak{g}}(\mu)=\lambda\left(f\left(\operatorname{ad}_{\mu}\right)\right)$ for $f(z)=\frac{1}{2} \operatorname{coth}(z / 2)-\frac{1}{z}$ solves the CDYBE for $\mathfrak{k}=\mathfrak{g}$, with coupling constant $\epsilon=\frac{1}{4}$. 
Proof. The proof relies on the identity (8) from Proposition 3.1. To simplify notation, we denote $\mathfrak{r}^{\mathfrak{g}}, \mathcal{S}^{\mathfrak{g}}$ simply by $\mathfrak{r}, \mathcal{S}$, respectively.

By Lemma 3.4, the right hand side of (8) is closed under the differential, $\delta^{\mathfrak{g}}+\sum_{a} \frac{\partial}{\partial \mu^{a}} \phi^{a}$. Hence

$$
\left(\delta^{\mathfrak{g}}+\sum_{a} \frac{\partial}{\partial \mu^{a}} \phi^{a}\right)\left(q \circ \iota_{\mathcal{S}}\left(e^{\lambda^{\mathfrak{g}}-\Sigma_{a} e_{a} \phi^{a}}\right)\right)=0 .
$$

By (12), this gives

$$
\left(\mathrm{d}^{\mathfrak{g}}+\frac{1}{4} \iota_{\Theta}+\sum_{a} \frac{\partial}{\partial \mu^{a}} \phi^{a}\right)\left(\iota_{\mathcal{S}}\left(e^{\lambda^{\mathfrak{g}}-\Sigma_{a} e_{a} \phi^{a}}\right)\right)=0 .
$$

Since $\iota_{\mathcal{S}}=J^{1 / 2} \exp \left(\iota_{\mathfrak{r}}\right)$ we have, using Lemma 3.3 ,

$$
\mathrm{d}^{\mathfrak{g}} \circ \iota_{\mathcal{S}}=\iota_{\mathcal{S}} \circ\left(\mathrm{d}^{\mathfrak{g}}-\iota_{\mathfrak{u}}+\sum_{a} e^{a} \circ \iota_{\left[e_{a}, \mathfrak{r}\right]} \mathfrak{g}-\frac{1}{2} \iota_{[\mathfrak{r}, \mathfrak{r}]} \mathfrak{g}\right)
$$

Furthermore,

$$
\frac{\partial}{\partial \mu^{a}} \circ \iota_{\mathcal{S}}=\iota_{\mathcal{S}} \circ\left(\frac{\partial}{\partial \mu^{a}}+\frac{1}{2} \frac{\partial \ln (J)}{\partial \mu^{a}}+\iota_{\frac{\partial \mathfrak{r}}{\partial \mu^{a}}}\right) .
$$

Using that $e^{\lambda^{\mathfrak{g}}-\sum_{a} e_{a} \phi^{a}}$ is closed under the differential $\mathrm{d}^{\mathfrak{g}}+\sum_{a} \frac{\partial}{\partial \mu^{a}} \phi^{a}$, we therefore obtain

$$
\begin{array}{r}
\left(-\iota_{\mathfrak{u}}+\sum_{a} e^{a} \circ \iota_{\left[e_{a}, \mathfrak{r}\right]} \mathfrak{g}-\frac{1}{2} \iota_{[\mathfrak{r}, \mathfrak{r}]^{\mathfrak{g}}}+\sum_{a}\left(\frac{1}{2} \frac{\partial \ln (J)}{\partial \mu^{a}}+\iota_{\frac{\partial \mathfrak{r}}{\partial \mu^{a}}}\right) \phi^{a}+\frac{1}{4} \iota_{\Theta}\right) \\
\left(e^{\lambda^{\mathfrak{g}}-\Sigma_{b} e_{b} \phi^{b}}\right)=0 .
\end{array}
$$

Multiply this Equation from the left by $\exp \left(\sum_{a} e_{a} \phi^{a}\right)$, and pick the coefficient cubic in $\phi$ 's. Only the three terms involving $\sum_{a} \phi^{a} \iota_{\frac{\partial \mathfrak{r}}{\partial \mu^{a}}}, \iota_{[\mathfrak{r}, \mathfrak{r}] \mathfrak{g}}$ and $\iota_{\Theta}$ contribute to this coefficient, and we obtain:

$$
\sum_{a} \phi\left(\frac{\partial \mathfrak{r}}{\partial \mu^{a}}\right) \wedge \phi^{a}+\frac{1}{2} \phi\left([\mathfrak{r}, \mathfrak{r}]^{\mathfrak{g}}\right)-\frac{1}{4} \phi(\Theta) \equiv \phi\left(\frac{\partial \mathfrak{r}}{\partial \mu^{a}} \wedge e^{a}+\frac{1}{2}[\mathfrak{r}, \mathfrak{r}]^{\mathfrak{g}}-\frac{1}{4} \Theta\right)=0 .
$$

Taking $E=\mathfrak{g}$, with $\phi$ the identity map, this is exactly the CDYBE.

Remark 4.2. It is not hard to work out the coefficients of $\phi$ of lower degree. Two of these identities simply state that $\mathfrak{r}$ is equivariant and $J$ is invariant. The remaining identity reads (cf. [3, Lemma A.2])

$$
\frac{1}{2} \sum_{a} \frac{\partial \ln (J)}{\partial \mu^{a}} e^{a}+\mathfrak{u}=0 .
$$

Theorem 4.1 was first obtained by Etingof-Varchenko [11] in the semi-simple case. See Etingof-Schiffmann [10] for a proof in the quadratic case.

More generally, let $\mathfrak{k} \subset \mathfrak{g}$ be a quadratic subalgebra of $\mathfrak{g}$, i.e. a subalgebra such that the restriction of $\mathcal{Q}$ to $\mathfrak{k}$ is non-degenerate. Let $\mathfrak{p}$ be the orthogonal complement of $\mathfrak{k}$, so that $\mathfrak{g}=\mathfrak{k} \oplus \mathfrak{p}$. Suppose that for $\mu$ in an open dense 
subset of $\mathfrak{k}$, the operator $\operatorname{ad}_{\mu}^{\mathfrak{p}}:=\left.\operatorname{ad}_{\mu}\right|_{\mathfrak{p}}$ is invertible. Let $\operatorname{ad}_{\mu}^{\mathfrak{k}}=\left.\operatorname{ad}_{\mu}\right|_{\mathfrak{k}}$ and define $\mathfrak{r}^{\mathfrak{k}}: \mathfrak{k} \rightarrow \wedge^{2} \mathfrak{k}$ and $\mathfrak{r}^{\mathfrak{p}}: \mathfrak{k} \rightarrow \wedge^{2} \mathfrak{p}$ by

$$
\mathfrak{r}^{\mathfrak{k}}(\mu)=\lambda\left(f\left(\operatorname{ad}_{\mu}^{\mathfrak{k}}\right)\right), \quad \mathfrak{r}^{\mathfrak{p}}(\mu)=\frac{1}{2} \lambda\left(\operatorname{coth}\left(\operatorname{ad}_{\mu}^{\mathfrak{p}} / 2\right)\right), \quad \mathfrak{r}=\mathfrak{r}^{\mathfrak{k}}+\mathfrak{r}^{\mathfrak{p}}
$$

Theorem 4.3. The function $\mathfrak{r}=\mathfrak{r}^{\mathfrak{k}}+\mathfrak{r}^{\mathfrak{p}}$ solves the $C D Y B E$ for $\mathfrak{k} \subset \mathfrak{g}$, with coupling constant $\epsilon=\frac{1}{4}$.

Proof. Let

$$
J^{\mathfrak{k}}(\mu)=\operatorname{det}\left(j\left(\operatorname{ad}_{\mu}^{\mathfrak{k}}\right)\right), \quad J^{\mathfrak{p}}(\mu)=\operatorname{det}\left(2 \sinh \left(\operatorname{ad}_{\mu}^{\mathfrak{p}} / 2\right)\right), \quad J=J^{\mathfrak{k}} J^{\mathfrak{p}},
$$

and

$$
\mathcal{S}^{\mathfrak{k}}=\left(J^{\mathfrak{k}}\right)^{1 / 2} \exp \left(\mathfrak{r}^{\mathfrak{k}}\right), \mathcal{S}^{\mathfrak{p}}=\left(J^{\mathfrak{p}}\right)^{1 / 2} \exp \left(\mathfrak{r}^{\mathfrak{p}}\right), \quad \mathcal{S}=\mathcal{S}^{\mathfrak{k}} \mathcal{S}^{\mathfrak{p}} .
$$

Here the square root $\left(J^{\mathfrak{p}}\right)^{1 / 2}$ is defined as a Pfaffian, for some choice of orientation on $\mathfrak{p}$. Let $d \mathrm{Vol}_{\mathfrak{p}}$ be the volume form defined by the orientation and the quadratic form $\left.\mathcal{Q}\right|_{\mathfrak{p}}$.

Let $e_{a}$ be a basis of $\mathfrak{g}$ given by a basis of $\mathfrak{k}$ followed by a basis of $\mathfrak{p}$. In what follows, summation over $a$ denotes summation over the entire basis, while summation over $i$ denotes summation over the basis of $\mathfrak{k}$.

The restriction of $\gamma^{\mathfrak{g}}$ to $\mathfrak{k}$ is a sum $\left.\gamma^{\mathfrak{g}}\right|_{\mathfrak{k}}=\gamma^{\mathfrak{k}}+\gamma^{\mathfrak{p}}$, where $\gamma^{\mathfrak{p}}$ takes values in $\mathrm{Cl}(\mathfrak{p})$. Combining Equation (8), with $\mathfrak{k}$ in place of $\mathfrak{g}$, with Equation (2), for $V=\mathfrak{p}$, we obtain the following identity in $C^{\infty}(\mathfrak{k}) \otimes \mathrm{Cl}(\mathfrak{g}) \otimes \wedge E$ :

$$
q \circ \iota_{\mathcal{S}}\left(e^{\lambda^{\mathfrak{k}}-\Sigma_{i} e_{i} \phi^{i}} \wedge \mathrm{d} \operatorname{Vol}_{\mathfrak{p}}\right)=e^{\left.\gamma^{\mathfrak{g}}\right|_{\mathfrak{k}}-\sum_{i} e_{i} \phi^{i}} .
$$

Write $\mathrm{d}^{\mathfrak{g}}=\mathrm{d}^{\mathfrak{k}}+\mathrm{d}^{\prime}$, where $\mathrm{d}^{\mathfrak{k}}$ is extended to $\wedge \mathfrak{g}$ by letting $\mathrm{d}^{\mathfrak{k}} \mu=0$ for $\mu \in \mathfrak{p}$. Since $\left.\gamma^{\mathfrak{g}}\right|_{\mathfrak{k}}-\sum_{i} e_{i} \phi^{i}$ is closed under the differential $\delta^{\mathfrak{g}}+\sum_{i} \frac{\partial}{\partial \mu^{i}} \phi^{i}$, and $\lambda^{\mathfrak{k}}-\sum_{i} e_{i} \phi^{i}$ is closed under the differential $\mathrm{d}^{\mathfrak{k}}+\sum_{i} \frac{\partial}{\partial \mu^{i}} \phi^{i}$ we can proceed as in the proof of Theorem 4.1 to obtain

$$
\begin{aligned}
\left(\mathrm{d}^{\prime}-\iota_{\mathfrak{u}}+\sum_{a} e^{a} \circ \iota_{\left[e_{a}, \mathfrak{r}\right]} \mathfrak{g}-\frac{1}{2} \iota[\mathfrak{r}, \mathfrak{r}]^{\mathfrak{g}}+\sum_{i}\left(\frac{1}{2} \frac{\partial \ln (J)}{\partial \mu^{i}}+\iota_{\frac{\partial \mathfrak{r}}{\partial \mu^{i}}}\right) \phi^{i}+\frac{1}{4} \iota_{\Theta}\right) & \\
\left(e^{\lambda^{\mathfrak{k}}-\Sigma_{i} e_{i} \phi^{i}}\right. & \left.\wedge \mathrm{d} \operatorname{Vol}_{\mathfrak{p}}\right)=0 .
\end{aligned}
$$

Multiply from the left by $e^{\sum_{a} e_{a} \phi^{a}-\lambda^{\mathfrak{k}}}$ (summation over the entire basis of $\mathfrak{g}$ ). The term cubic in $\phi$ 's is proportional to $d \operatorname{Vol}_{\mathfrak{p}}$, and the coefficient gives the CDYBE.

Still more generally, suppose $c \in \mathrm{O}(\mathfrak{g})$ is an automorphism of $\mathfrak{g}$. Suppose $\mathfrak{k}$ is a quadratic subalgebra contained in the fixed point set of $c$. Let $\mathfrak{p}$ be its orthogonal complement as above, and suppose that for $\mu$ in an open dense subset of $\mathfrak{k}$, the operator $c \exp \left(\operatorname{ad}_{\mu}\right)-I$ is invertible on $\mathfrak{p}$. Then

$J_{c}^{\mathfrak{p}}=\operatorname{det}_{\mathfrak{p}}\left(c \exp \left(\operatorname{ad}_{\mu}^{\mathfrak{p}}\right)-I\right), \quad \mathfrak{r}_{c}^{\mathfrak{p}}=\frac{1}{2} \lambda\left(\left.\frac{c \exp \left(\operatorname{ad}_{\mu}\right)+I}{c \exp \left(\operatorname{ad}_{\mu}\right)-I}\right|_{\mathfrak{p}}\right), \quad \mathcal{S}_{c}^{\mathfrak{p}}=\left(J_{c}^{\mathfrak{p}}\right)^{1 / 2} \exp \left(\mathfrak{r}_{c}^{\mathfrak{p}}\right)$

are well-defined meromorphic functions on $\mathfrak{k}$. 
Theorem 4.4. The function $\mathfrak{r}=\mathfrak{r}^{\mathfrak{k}}+\mathfrak{r}_{c}^{\mathfrak{p}}$ solves the CDYBE with coupling constant $\epsilon=\frac{1}{4}$.

Proof. Equations (3) and (8) give the following identity in $C^{\infty}(\mathfrak{k}) \otimes \mathrm{Cl}(\mathfrak{g}) \otimes \wedge E$ :

$$
q \circ \iota_{\mathcal{S}}\left(e^{\lambda^{\mathfrak{k}}-\sum_{i} e_{i} \phi^{i}} \wedge \mathrm{d} \operatorname{Vol}_{\mathfrak{p}}\right)= \pm \hat{c} e^{\left.\gamma^{\mathfrak{g}}\right|_{\mathfrak{k}}-\sum_{i} e_{i} \phi^{i}}
$$

where $\mathcal{S}=\mathcal{S}^{\mathfrak{k}} \mathcal{S}_{c}^{\mathfrak{p}}$. The element $\widehat{c}$ commutes with $q(\Theta)$, since $c$ is an automorphism of $\mathfrak{g}$ preserving the quadratic form. Hence $\widehat{c}$ commutes with $\delta^{\mathfrak{g}}$, and hence the right hand side of (15) is closed under $\delta^{\mathfrak{g}}+\sum_{i} \frac{\partial}{\partial \mu^{i}} \phi^{i}$. The rest of the proof is as before.

The classical dynamical r-matrix described here was first obtained by EtingofSchiffmann [10], for the case that $c$ is a finite order automorphism and $\mathfrak{k}=\mathfrak{g}_{0}$. (Note that the fixed point set of a finite order automorphism $c \in O(\mathfrak{g})$ is a quadratic subalgebra.)

Example 4.5. Let $\left(\mathfrak{k}, \mathcal{Q}^{\mathfrak{k}}\right)$ be a quadratic Lie algebra, and $\mathfrak{g}=\mathfrak{k}^{\mathbb{C}}$ its complexification. The real part of the complexification of $\mathcal{Q}^{\mathfrak{k}}$ defines a non-degenerate quadratic form $\mathcal{Q}$ on $\mathfrak{g}$, with $\mathfrak{p}=\sqrt{-1} \mathfrak{k}$ (viewed as a real subalgebra). Let $c \in \mathrm{O}(\mathfrak{g})$ denote the automorphism given by complex conjugation. The $\mathfrak{r}$-matrix described above has the form

$$
\mathfrak{r}=\lambda\left(f\left(\operatorname{ad}_{\mu}^{\mathfrak{k}}\right)+\frac{1}{2} \tanh \left(\operatorname{ad}_{\mu}^{\mathfrak{p}} / 2\right)\right) .
$$

As explained in $[11,9]$, one obtains other solutions of the CDYBE by scaling or taking limits:

(i) If $\mathfrak{r}$ is a solution of the CDYBE for coupling constant $\epsilon$, then $\mathfrak{r}_{t}(\mu):=$ $t^{-1} \mathfrak{r}\left(t^{-1} \mu\right)$ is a solution with coupling constant $t^{-2} \epsilon$. Applying this to the trigonometric solutions obtained above, and taking the limit for $t \rightarrow \infty$ one obtains rational solutions of the CDYBE with vanishing coupling constant. If $\mathfrak{r}$ is anti-symmetric in $\mu$, one can also take imaginary $t$ changing the sign of the coupling constant. This replaces coth with cot in our formulas.

(ii) For any element $\nu$ in the center of $\mathfrak{k}$, the shifted $r$-matrix $\mu \mapsto \mathfrak{r}(\mu+\nu)$ again solves the CDYBE for coupling constant $\epsilon$. Furthermore, if the limit

$$
\mathfrak{r}_{\nu}(\mu)=\lim _{t \rightarrow \infty} \mathfrak{r}(\mu+t \nu)
$$

exists, then the limiting $r$-matrix again solves the CDYBE for $\epsilon$. For instance, if $\mathfrak{g}$ is semi-simple and $\mathfrak{k}=\mathfrak{t}$, one obtains constant $r$-matrices by taking $\nu \in \mathfrak{t}$ some regular element.

(iii) Recall that classical dynamical $r$-matrices $\mathfrak{r}: \mathfrak{k} \rightarrow \wedge^{2} \mathfrak{g}$ are always required to be $\mathfrak{k}$-invariant. Hence, the Schouten bracket of any element of $\wedge \mathfrak{k}$ with $\mathfrak{r}$ vanishes. Thus if $\mathfrak{s}: \mathfrak{k} \rightarrow \wedge^{2} \mathfrak{k}$ solves the CDYBE for $(\mathfrak{k}, \mathfrak{k})$ with coupling constant $\delta$, then $\mathfrak{r}+\mathfrak{s}$ solves the CDYBE for $(\mathfrak{g}, \mathfrak{k})$ with coupling constant $\delta+\epsilon$. In particular, if $\mathfrak{k}$ is Abelian, any closed 2 -form on $\mathfrak{k}$ gives rise to a solution of the CDYBE for $(\mathfrak{k}, \mathfrak{k})$ with coupling constant 0 . 


\section{Clifford algebra calculations}

In this section we prove the Clifford algebra identities from Section 2. These formulas are most systematically obtained from the spinor representation for the Clifford algebra of the direct sum $V \oplus V^{*}$, which we briefly review.

5.1. Spinor representation. Let $V$ be a finite-dimensional real vector space. The direct sum $W=V \oplus V^{*}$ carries a quadratic form

$$
\mathcal{Q}_{W}(v \oplus \alpha)=2 \alpha(v) .
$$

Let $\mathrm{Cl}(W)$ be the Clifford algebra of $\left(W, \mathcal{Q}_{W}\right)$, and consider the algebra representation

$$
\pi: \mathrm{Cl}(W) \rightarrow \mathfrak{g l}(\wedge V)
$$

where generators $v \in V$ act by wedge product and generators $\alpha \in V^{*}$ act by contraction. The restriction of $\pi$ to a group representation of $\operatorname{Spin}(W) \subset$ $\mathrm{Cl}(W)^{\times}$is called the spinor representation.

The group $\mathrm{SO}(W)$ contains $\wedge^{2}(V), \wedge^{2}\left(V^{*}\right), \mathrm{GL}(V)$ as distinguished subgroups, lifting to subgroups $\wedge^{2}(V), \wedge^{2}\left(V^{*}\right), \operatorname{ML}(V)$ of $\operatorname{Spin}(W)$ :

(i) For any skew-adjoint linear map $D: V^{*} \rightarrow V$ let $\lambda(D)=\frac{1}{2} \sum_{a} D\left(e^{a}\right) \wedge e_{a} \in$ $\wedge^{2}(V)$. There is an inclusion

$$
\wedge^{2}(V) \rightarrow \mathrm{SO}(W), \quad \lambda(D) \mapsto\left(\begin{array}{cc}
I & D \\
0 & I
\end{array}\right)
$$

This inclusion lifts to a vector subgroup $\wedge^{2}(V) \hookrightarrow \operatorname{Spin}(W)$, and the action of $\pi(\lambda(D))$ is wedge product with $\exp (\lambda(D))$.

(ii) Similarly, there is an identification $E \mapsto \lambda(E)$ of skew-adjoint linear maps $V \rightarrow V^{*}$ with $\wedge^{2} V^{*}$. The inclusion

$$
\wedge^{2} V^{*} \rightarrow \mathrm{SO}(W), \lambda(E) \mapsto\left(\begin{array}{cc}
I & 0 \\
E & I
\end{array}\right) .
$$

lifts to an inclusion $\wedge^{2} V^{*} \hookrightarrow \operatorname{Spin}(W)$, and $\pi(\lambda(E))$ is given by contraction with $\exp (\lambda(E))$.

(iii) Finally, there is an inclusion

$$
\mathrm{GL}(V) \rightarrow \mathrm{SO}(W), \quad R \mapsto\left(\begin{array}{cc}
R & 0 \\
0 & \left(R^{-1}\right)^{*}
\end{array}\right)
$$

The metalinear group $\mathrm{ML}(V) \hookrightarrow \operatorname{Spin}(W)$ is the inverse image of $\mathrm{GL}(V)$ under the covering map $\operatorname{Spin}(W) \rightarrow \mathrm{SO}(W)$. The action of an element $\hat{R} \in \mathrm{ML}(V)$, covering $R \in \mathrm{GL}(V)$, in the spinor representation is given by

$$
\pi(\hat{R}) \cdot \alpha=\frac{R \cdot \alpha}{|\operatorname{det}|^{1 / 2}(\hat{R})} .
$$

Here $|\operatorname{det}|^{1 / 2}: \operatorname{ML}(V) \rightarrow \mathbb{R}^{\times}$is a suitable choice of square root of $|\operatorname{det}|$ : $\mathrm{GL}(V) \rightarrow \mathbb{R}_{>0}$ (defined by this formula), and $R . \alpha$ is defined by the unique extension of $R \in \mathrm{GL}(V)$ to an algebra automorphism of $\wedge(V)$. 
5.2. The action of $\operatorname{Spin}(V)$ on $\wedge V$. We now return to our original setting, where $V$ itself comes equipped with a quadratic form $\mathcal{Q}_{V}$. Under the identification $q: \wedge V \cong \mathrm{Cl}(V)$, the left multiplication of the Clifford algebra on itself defines a representation,

$$
\varrho: \mathrm{Cl}(V) \rightarrow \mathfrak{g l}(\wedge V),
$$

given on generators $v \in V$ by

$$
\varrho(v) . \alpha=v \wedge \alpha+\frac{1}{2} \iota_{v} \alpha .
$$

The symbol map $q^{-1}: \mathrm{Cl}(V) \rightarrow \wedge(V)$ can be expressed in terms of $\varrho$ as $q^{-1}(x)=$ $\varrho(x) .1$, the action on $1 \in \wedge V$.

We will now relate $\varrho$ (hence also the symbol map) to the representation $\pi$ from 5.1. Let $\bar{V}$ denote the same vector space with quadratic form $\mathcal{Q}_{\bar{V}}=-\mathcal{Q}_{V}$. Then

$$
\kappa: V \oplus \bar{V} \rightarrow W, \quad(v, w) \mapsto\left(v+w, \frac{1}{2}(v-w)\right)
$$

is an isometry, with inverse $\kappa^{-1}(x, y)=(x / 2+y, x / 2-y)$. Using the isomorphism

$$
\mathrm{Cl}(V) \otimes \mathrm{Cl}(\bar{V})=\mathrm{Cl}(W)
$$

to view $\mathrm{Cl}(V)$ as a subalgebra of $\mathrm{Cl}(W)$, the homomorphism $\varrho: \operatorname{Cl}(V) \rightarrow \mathfrak{g l}(\wedge V)$ is simply the restriction of $\pi$. The inclusion $\mathrm{Cl}(V) \rightarrow \mathrm{Cl}(W)$ restricts to an inclusion of Spin groups, $\operatorname{Spin}(V) \rightarrow \operatorname{Spin}(W)$. The corresponding inclusion $\iota: \mathrm{SO}(V) \rightarrow \mathrm{SO}(W)$ is given by

$$
\iota: \mathrm{SO}(V) \rightarrow \mathrm{SO}(W), \quad C \mapsto \kappa \circ\left(\begin{array}{cc}
C & 0 \\
0 & I
\end{array}\right) \circ \kappa^{-1}=\left(\begin{array}{cc}
\frac{1}{2}(C+I) & C-I \\
\frac{1}{4}(C-I) & \frac{1}{2}(C+I)
\end{array}\right) .
$$

Proposition 5.1. Let $C \in \mathrm{SO}(V)$ with $\operatorname{det}(C-I) \neq 0$, and suppose that $D \in$ $\mathrm{o}(V)$ is invertible and commutes with $C$. Then there is a unique factorization

$$
\iota(C)=\left(\begin{array}{cc}
I & 0 \\
E_{1} & I
\end{array}\right)\left(\begin{array}{cc}
I & D \\
0 & I
\end{array}\right)\left(\begin{array}{cc}
I & 0 \\
E_{2} & I
\end{array}\right)\left(\begin{array}{cc}
R & 0 \\
0 & \left(R^{-1}\right)^{t}
\end{array}\right)
$$

such that $E_{1}, E_{2} \in \mathrm{o}(V)$ and $R \in \mathrm{GL}(V)$ commute with $C$ and $D$. One finds

$$
E_{1}=\frac{1}{2} \frac{C+I}{C-I}-\frac{1}{D}, \quad E_{2}=\frac{1}{D^{2}}\left(\frac{C-C^{-1}}{2}-D\right), \quad R=\frac{D}{I-C^{-1}} .
$$

Proof. Working out the matrix product on the right hand side Equation (18) reads

$$
\left(\begin{array}{cc}
\frac{1}{2}(C+I) & C-I \\
\frac{1}{4}(C-I) & \frac{1}{2}(C+I)
\end{array}\right)=\left(\begin{array}{cc}
\left(I+D E_{2}\right) R & D\left(R^{-1}\right)^{t} \\
\left(E_{1}+E_{1} D E_{2}+E_{2}\right) R & \left(I+D E_{1}\right)\left(R^{-1}\right)^{t}
\end{array}\right)
$$

It is straightforward to check this equality with the given formulas for $R, E_{1}, E_{2}$. Conversely, equality of the upper right corners gives $R$, and then the diagonal entries give our formulas for $E_{1}, E_{2}$. 
The factorization (18) gives rise to a factorization for any $\hat{C} \in \operatorname{Spin}(V)$ covering $C$. The first three factors lift as in (i),(ii) above, and a lift $\widehat{R} \in \operatorname{ML}(V)$ of $R=D /\left(I-C^{-1}\right)$ is determined by the choice of lift $\hat{C}$ of $C$. Using the known action of each factor in the representation $\pi$ we obtain:

Proposition 5.2. Suppose $\hat{C} \in \operatorname{Spin}(V)$ maps to $C \in \mathrm{SO}(V)$ with $\operatorname{det}(C-I) \neq$ 0 , and that $D \in \mathrm{o}(V)$ is invertible and commutes with $C$. Let $E_{1}, E_{2} \in \mathrm{o}(V)$ and $\hat{R} \in \mathrm{ML}^{+}(V)$ be as above. Then the operator $\varrho(\hat{C})$ on $\wedge V$ has the following factorization:

$$
\varrho(\hat{C}) \cdot \alpha=\frac{\exp \left(\iota_{\lambda\left(E_{1}\right)}\right) \exp (\lambda(D)) \exp \left(\iota_{\lambda\left(E_{2}\right)}\right) R \cdot \alpha}{|\operatorname{det}|^{1 / 2}(\hat{R})} .
$$

In particular, the symbol of $\hat{C}$ is given by the formula,

$$
q^{-1}(\hat{C})=\frac{\exp \left(\iota_{\lambda\left(E_{1}\right)}\right) \exp (\lambda(D))}{|\operatorname{det}|^{1 / 2}(\hat{R})} .
$$

Given $\hat{C}$, there may be many natural choices of $D$ with the required properties, leading to different formulas for the symbol of $\hat{C}$.

\section{Proposition 5.3.}

a. Suppose $\hat{C} \in \operatorname{Spin}(V)$ maps to $C \in \mathrm{SO}(V)$, with $\operatorname{det}(C+I) \neq 0$. Then

$$
q^{-1}(\hat{C})= \pm \operatorname{det}^{1 / 2}\left(\frac{C+I}{2}\right) \exp \left(2 \lambda\left(\frac{C-I}{C+I}\right)\right) .
$$

b. Suppose $\hat{C} \in \operatorname{Pin}(V)$ maps to $C \in \mathrm{O}(V)$, with $\operatorname{det}(C-I) \neq 0$. Then

$$
q^{-1}(\hat{C})= \pm \operatorname{det}^{1 / 2}\left(I-C^{-1}\right) \exp \left(\iota_{\lambda\left(\frac{1}{2} \frac{C+I}{C-I}\right)}\right) d \mathrm{Vol},
$$

where $d \mathrm{Vol}$ is the volume form on $V$ given by the quadratic form and some choice of orientation.

Proof. Let us first assume that $\hat{C} \in \operatorname{Spin}(V)$ and that $\operatorname{both} \operatorname{det}(C+I) \neq 0$ and $\operatorname{det}(C-I) \neq 0$. (In particular, $\operatorname{dim} V$ must be even.) The first formula is obtained from the choice

$$
D=2(C-I) /(C+I),
$$

since $E_{1}=0$ in this case. Let $D_{t}:=t D$ for $t>0$. As $t \rightarrow \infty$,

$$
\exp \left(\lambda\left(D_{t}\right)\right)=t^{\operatorname{dim} V / 2} \operatorname{det}^{1 / 2}(D) \mathrm{d} \operatorname{Vol}+O\left(t^{\operatorname{dim} V / 2-1}\right),
$$

where $\operatorname{det}^{1 / 2}(D)$ the Pfaffian corresponding to the choice of orientation, and

$$
\operatorname{det}^{1 / 2}\left(R_{t}\right)=t^{\operatorname{dim} V / 2} \operatorname{det}^{1 / 2}\left(\frac{D}{I-C^{-1}}\right) .
$$

The factors $t^{\operatorname{dim} V / 2} \operatorname{det}^{1 / 2}(D)$ cancel, and taking the limit $t \rightarrow \infty$ we obtain the second formula. By continuity, one can drop the assumption $\operatorname{det}(C-I) \neq 0$ in the first formula and the assumption $\operatorname{det}(C+I) \neq 0$ in the second formula. The first formula also holds if $\operatorname{dim} V$ is odd, by restricting the formula for $V \oplus \mathbb{R}$. Similarly, to extend the second formula to $\widehat{C} \in \operatorname{Pin}(V)$ one replaces $V$ with $V \oplus \mathbb{R}$ and $C$ with $\left(\begin{array}{cc}C & 0 \\ 0 & -1\end{array}\right)$. 
The sign ambiguity is resolved if $C=\exp (A)$ and $\hat{C}=\exp (\gamma(A))$. In this case, Equation (21) reduces to (1) and Equation (22) to (2). Similarly, Equation (3) is obtained from $(22)$ by the choice $C=c \exp (A)$. The function $\mathcal{S}$ appears if we restate (19) for $\hat{C}=\exp (\gamma(A))$ and the choice $D=A$. For the time being, we treat $\mathcal{S}$ as a meromorphic function of $A$.

Proposition 5.4. Suppose $A \in \mathrm{o}(V)$ has no eigenvalues in the set $2 \pi \sqrt{-1} \mathbb{Z} \backslash\{0\}$. Then the operator $\varrho(\exp (\gamma(A)))$ on $\wedge V$ has the following factorization:

$$
\varrho(\exp (\gamma(A)))=\iota_{\mathcal{S}(A)} \circ \exp (\lambda(A)) \circ \exp \left(\iota_{\lambda(g(A))}\right) \circ j^{L}(A)^{-1} .
$$

In particular,

$$
q^{-1}(\exp (\gamma(A)))=\iota_{\mathcal{S}(A)} \exp (\lambda(A))
$$

Proof. The assumption on $A$ implies that $f(A), g(A), j^{L}(A), j^{R}(A)$ are all welldefined and that $j^{L}(A)$ is invertible. If $\operatorname{dim} V$ is even, the two sides of $(24)$ are equal since they agree on the open dense subset where $A$ is invertible, by Proposition 5.2. The odd-dimensional case follows by restricting the identity for $V \oplus \mathbb{R}$.

Equation (25) gives Theorem 2.1 for $\phi=\{0\}$. We now show that in fact, it implies the general case.

Proof of Theorem 2.1. Let $\tilde{V}=V \oplus E$. Fix a non-degenerate quadratic form $\mathcal{Q}_{E}$ on $E$, and let $\tilde{V}$ be equipped with the quadratic form $\mathcal{Q}_{\tilde{V}}=\mathcal{Q}_{V} \oplus \epsilon \mathcal{Q}_{E}$ for $\epsilon>0$. Then $\lambda(A)-\sum_{a} e_{a} \phi^{a}=\lambda(\tilde{A})$ with

$$
\tilde{A}=\left(\begin{array}{cc}
A & -\epsilon \phi^{t} \\
\epsilon \phi & 0
\end{array}\right) \in \mathrm{o}(\tilde{V})
$$

By the above, $q \circ \iota_{\mathcal{S}(\tilde{A})} \exp (\lambda(\tilde{A}))=\exp (\gamma(\tilde{A}))$. Equation (6) follows by letting $\epsilon \rightarrow 0$. It remains to show that $\mathcal{S}$ is analytic everywhere. Equation (6) says that

$$
\iota_{\mathcal{S}(A)} \alpha(A)=\beta(A)
$$

where $\alpha(A)=e^{\lambda(A)-\Sigma_{a} e_{a} \phi^{a}}$ and $\beta(A)=q^{-1}\left(e^{\gamma(A)-\Sigma_{a} e_{a} \phi^{a}}\right)$ are differential forms depending analytically on $A$. Take $E=V$ and $\phi=$ id. Then $-\sum_{a} e_{a} \phi^{a} \in$ $\wedge^{2}(V \oplus E)$ is non-degenerate. Equivalently, the form $\Gamma$ given as the top degree part of $\exp \left(-\sum_{a} e_{a} \phi^{a}\right)$ is a volume form. Let $*$ denote the star operator defined by $\Gamma$, i.e. $\zeta=* \iota_{\zeta} \Gamma$ for $\zeta \in \wedge(V \oplus E)$. Then $\iota_{\mathcal{S}(A)} \alpha(A)=\beta(A)$ is equivalent to $\mathcal{S}(A) \wedge * \alpha(A)=* \beta(A)$. Since the top form degree part of $\alpha(A)$ coincides with $\Gamma$, the constant term of $* \alpha(A)$ is equal to 1 . Hence $* \alpha(A)^{-1}$ is well-defined and depends analytically on $A$. We obtain

$$
\mathcal{S}(A)=* \beta(A) \wedge(* \alpha(A))^{-1},
$$

showing explicitly that $\mathcal{S}(A)$ is analytic everywhere.

As another application of the factorization formula (24) we prove the alternative formula (7) for the Clifford exponential: 
Proof of (7). Apply Equation (24) to $\alpha=\exp \left(-\sum_{a} e_{a} \psi^{a}\right) \in \wedge(V) \otimes \wedge(E)$. The left hand side is

$$
q^{-1}\left(\exp (\gamma(A)) \exp \left(-\sum_{a} e_{a} \psi^{a}\right)\right)
$$

To compute the right hand side, we first note that

$$
j^{L}(A)^{-1} \sum_{a} e_{a} \psi^{a}=\sum_{a}\left(j^{L}(A)^{-1} \cdot e_{a}\right) \phi\left(j^{R}(A) e^{a}\right)=\sum_{a} e_{a} \phi^{a} .
$$

Hence $j^{L}(A)^{-1} \cdot \exp \left(-\sum_{a} e_{a} \psi^{a}\right)=\exp \left(-\sum_{a} e_{a} \phi^{a}\right)$. Furthermore,

$$
\exp \left(\iota_{\lambda(g(A))}\right) \cdot \exp \left(-\sum_{a} e_{a} \phi^{a}\right)=\exp (\varpi(A)) \exp \left(-\sum_{a} e_{a} \phi^{a}\right) .
$$

Hence the right hand side of (24) becomes

$$
\exp (\varpi(A)) \iota_{\mathcal{S}(A)}\left(\exp (\lambda(A)) \exp \left(-\sum_{a} e_{a} \phi^{a}\right)\right)
$$

\section{References}

[1] A. Alekseev, Y. Kosmann-Schwarzbach, E. Meinrenken, Quasi-Poisson manifolds, Canad. J. Math. 54 (2002), 3-29.

[2] A. Alekseev, A. Malkin, E. Meinrenken, Lie group valued moment maps, J. Differential Geom. 48 (1998), 445-495.

[3] A. Alekseev, E. Meinrenken, The non-commutative Weil algebra, Invent. Math. 139 (2000), 135-172.

[4] J. Balog, L. Dạbrowski, and L. Fehér, Classical $r$-matrix and exchange algebra in WZNW and Toda theories, Phys. Lett. B 244 (1990), 227-234.

[5] J. Balog, L. Fehér, and L. Palla, Chiral extensions of the WZNW phase space, Poisson-Lie symmetries and groupoids, Nuclear Phys. B 568 (2000), 503-542.

[6] A. A. Belavin, V. G. Drinfel'd, Triangle equations and simple Lie algebras, Mathematical physics reviews, Vol. 4, 93-165, Harwood Academic Publ., Chur, 1984.

[7] N. Berline, E. Getzler, M. Vergne, Heat kernels and Dirac operators, Grundlehren der Mathematischen Wissenschaften, 298. Springer-Verlag, Berlin, 1992.

[8] V.G. Drinfel'd, Hamiltonian structures on Lie groups, Lie bialgebras and the geometric meaning of classical Yang-Baxter equations, Dokl. Akad. Nauk SSSR 268 (1983), 285287.

[9] P. Etingof, O. Schiffmann, Lectures on the classical dynamical Yang-Baxter equations, preprint, 1999.

[10] $157-170$.

[11] P. Etingof, A. Varchenko, Geometry and classification of solutions of the classical dynamical Yang-Baxter equation, Comm. Math. Phys. 192 (1998), 77-120.

[12] L. Fehér, A. Gábor, B. G. Pusztai, On dynamical $r$-matrices obtained from Dirac reduction and their generalizations to affine Lie algebras, J. Phys. A 34 (2001), 7335-7348.

[13] G. Felder, Conformal field theory and integrable systems associated to elliptic curves, Proceedings of the International Congress of Mathematicians, Vol. 1, 2 (Zürich, 1994), 1247-1255, Birkhäuser, Basel, 1995.

[14] S. Helgason, Differential geometry, Lie groups, and symmetric spaces, Pure and Applied Mathematics, 80. Academic Press, Inc., New York-London, 1978. 
[15] B. Kostant, S. Sternberg, Symplectic reduction, BRS cohomology, and infinitedimensional Clifford algebras, Ann. Physics 176 (1987), 49-113.

[16] J.-H. Lu, Classical dynamical r-matrices and homogeneous Poisson structures on $G / H$ and $K / T$, Comm. Math. Phys. 212 (2000), 337-370.

[17] A. Medina, P. Revoy, Algèbres de Lie et produit scalaire invariant, Ann. Sci. École Norm. Sup. (4) 18 (1985), 553-561.

[18] E. Petracci, PhD thesis, in preparation.

[19] O. Schiffmann, On classification of dynamical r-matrices, Math. Res. Lett. 5 (1998), 13-30.

[20] M. A. Semenov-Tian-Shansky, What is a classical r-matrix?, Funct. Anal. Appl. 17 (1983), 259-272.

[21] P. Xu, Quantum dynamical Yang-Baxter equation over non-abelian base, preprint, 2001.

University of Geneva, Section of Mathematics, 2-4 Rue du Lièvre, 1211 Genève 24, SWITZERLAND.

E-mail address: alekseev@math.unige.ch

University of Toronto, Department of Mathematics, 100 St George Street, Toronto, Ontario M5S3G3, Canada.

E-mail address: mein@math.toronto.edu 